\title{
Nano-size Effect of Interface Energy and Its Effect on Interface Fracture
}

\author{
L. H. Liang ${ }^{\mathrm{a}}{ }^{*}$, X. M. You ${ }^{\mathrm{a}}$, H. S. Ma ${ }^{\mathrm{a}}$, and Y. G. Wei ${ }^{\mathrm{a}}$ \\ ${ }^{a}$ LNM, Institute of Mechanics, Chinese Academy of Sciences, Beijing 100190, China (*lianglh@lnm.imech.ac.cn)
}

\begin{abstract}
An analytical model about size-dependent interface energy of metal/ceramic interfaces in nanoscale is developed by introducing both the chemical energy and the structure stain energy contributions. The dependence of interface energy on the interface thickness is determined by the melting enthalpy, the molar volume, and the shear modulus of two materials composing the interfaces, etc. The analytic prediction of the interface energy and the atomic scale simulation of the interface fracture strength are compared with each other for $\mathrm{Ag} / \mathrm{MgO}$ and $\mathrm{Ni} / \mathrm{Al}_{2} \mathrm{O}_{3}$ interfaces, the fracture strength of the interface with the lower chemical interface energy is found to be larger. The potential of $\mathrm{Ag} / \mathrm{MgO}$ interface related to the interface energy is calculated, and the interface stress and the interface fracture strength are estimated further. The effect of the interface energy on the interface strength and the behind mechanism are discussed.
\end{abstract}

Keywords: Interface Energy, Size Effect, Fracture Strength.

PACS: $68.35 . \mathrm{Gy}, 62.25 .+\mathrm{g}, 62.20 . \mathrm{Mk}$

\section{INTRODUCTION}

Interface energy study has attracted great attention in both experimental and theoretical aspects due to its importance in extensive fields. The stability of interface determined by the interface energy affects the performance of materials, structures and devices. Specially, the properties of metal/ceramic interfaces play important role in thermal barrier coatings used in aircraft and power generation turbines. Metal/ceramic interfaces have also extensive applications in catalytic converters, field effect transistors, and anticorrosion coatings, etc. The instability of the interface may lead to the lapse of materials and devices. Especially, the fracture is severe interface failure way, the fracture strength and the toughness are important parameters determining the interface fracture properties and are closely related to the interface energy. Experimental measurement of the interface energy is difficult due to the heat conduction change of samples and vessels. The theoretical calculation based on the first principle, the embedded atomic method and the molecular dynamic is complicated and time-consuming. Therefore, the scientific theoretical prediction of the interface energy is significant. The size effect of the interface energy can not be neglected with the application of low-dimensional materials and the development of MEMS and NEMS. Study has found that the solid-solid interface energy and the solid-liquid energy of single materials are size-dependent [1], the size effect is obvious in nanoscale, which affects the phase transition of materials. The interface energy of different materials or composite materials is also size-dependent [2], which will affect the adhesion property and the stability of the interface structures. Therefore, to study nanometer size effect of the interface energy and its effect on the interface fracture is in great interest.

In this paper, an analytical model of size-dependent interface energy is developed, the factors determining the size effect are discovered. Further, the interface energy and the interface fracture strength of two metal/ceramic systems are compared with each other, and the relation between two interface quantities is discussed 


\section{MODEL}

The interface energy $\gamma$ is the excess energy per unit area of a system due to appearing of the interface, which is originated from the change of the interfacial atomic bonding refereed to be as the chemical interface energy $\gamma_{c}$ and the structure stain at the interface refereed to be as the structural interface energy $\gamma_{\mathrm{s}}$. The chemical interface energy makes main contribution to the metal/ceramic interfaces considering the greater chemical component difference of both materials, which is related to the intrinsic solid-solid interface energies of metals and ceramics $\gamma_{\mathrm{cl}}$ and $\gamma_{\mathrm{c} 2}$, thus $\gamma_{\mathrm{c}}$ is taken as the average value of $\gamma_{\mathrm{c} 1}$ and $\gamma_{\mathrm{c} 2}$. The size effect of the interface energy should be considered at the nanometer scale, the size-dependent intrinsic solid-solid interface energy has been derived as [1]

$$
\gamma_{\mathrm{ci}}=\gamma_{\mathrm{bi}}\left[1-D_{0 \mathrm{i}} /(4 D)\right]
$$

where $\mathrm{i}=1,2$ denotes the metal and the ceramic, respectively, $\gamma_{\mathrm{b}}=2 \gamma_{\mathrm{m}}$ is the corresponding bulk solid-solid interface energy with the corresponding bulk solid-liquid interface energy $\gamma_{\mathrm{m}}$ [3], $D_{0}=2 h$ is the critical size with the atomic diameter $h$ [1], and $D=t_{1}+t_{2}$ is the thickness of the interface with the thickness of metals and ceramics $t_{1}$ and $t_{2}$. The solid-liquid interface energy $\gamma_{\mathrm{m}}=2 h S H /(3 V R)$, where $S=H / T$ is the melting entropy with the melting enthalpy $H$ and the melting temperature $T, V$ is the molar volume of crystals, and $R$ is the ideal gas constant [1]. Therefore, the size-dependent chemical interface energy is expressed as

$$
\gamma_{\mathrm{c}}=2\left\{h_{1} S_{1} H_{1}\left[1-h_{1} /(2 D)\right] / V_{1}+h_{2} S_{2} H_{2}\left[1-h_{2} /(2 D)\right] / V_{2}\right\} /(3 R) .
$$

Equation (2) shows that the chemical interface energy is size-dependent and the size effect is related to some available thermodynamic quantities. On the other hand, the structure interface energy caused by mismatch and dislocation strain should be considered. Assuming the semi-coherent interface is formed between metals and ceramics, the structure interface energy is expressed as [4]

$$
\gamma_{\mathrm{s}}=C b \varepsilon_{0}\{\ln [D /(2 b)]+1\}\left(1-\varepsilon / \varepsilon_{0}\right) /(2 \pi),
$$

where $C=\left\{2\left[\left(1-v_{1}\right) / G_{1}+\left(1-v_{2}\right) / G_{2}\right]\right\}^{-1}$ is the effective elastic modulus with the Passion's ratio $v$ and the shear modulus $G, b=\left(h_{1}+h_{2}\right) / 2$ is the Burgers vector, $\varepsilon_{0}=\left(h_{2}-h_{1}\right) / h_{1}$ is the misfit strain, $D / 2$ represents the effective dislocation stress-field radius, $\varepsilon$ is the in-plane strain of the thin layer composing the interface with respect to its bulk state and is determined by [5]

$$
\varepsilon=-2 f(1-v) /(E D),
$$

where $f$ is the intrinsic interface stress, $E$ is the Young's modulus, $E$ and $v$ are both taken as the average values of metals and ceramics. The intrinsic interface stress $f$ is calculated by $f=-\left[\left(3 \gamma_{\mathrm{m}} D_{0}\right) /(8 \kappa)\right]^{1 / 2}$, where $\kappa=1 / B$ is the compressibility with the volume modulus $B$ [5], $\gamma_{\mathrm{m}}, D_{0}$ and $\kappa$ are also taken as the average values of metals and ceramics, the negative sign shows the interface stress is compressive. Substituting Eq. (4) into Eq. (3), the size-dependent structural interface energy can be obtained. Finally, combining Eqs. (2) and (3), the total interface energy of $\gamma$ is expressed as

$$
\gamma=\gamma_{\mathrm{c}}+\gamma_{\mathrm{s}}
$$

\section{DISCUSSIONS}

Figure 1 shows the predicted interface energy of $\mathrm{Ag} / \mathrm{MgO}$ and $\mathrm{Ni} / \mathrm{Al}_{2} \mathrm{O}_{3}$ interfaces based on the above model. It

can be seen from the figure that the interface energy decreases obviously with reducing size at nanometer scale, and the interface energy of $\mathrm{Ag} / \mathrm{MgO}$ is lower than that of $\mathrm{Ni} / \mathrm{Al}_{2} \mathrm{O}_{3}$ at the same interface thickness. However, according to Eq. (2), the chemical interface energy of $\mathrm{Ag} / \mathrm{MgO}$ is higher than that of $\mathrm{Ni} / \mathrm{Al}_{2} \mathrm{O}_{3}$, thus the structural strain 

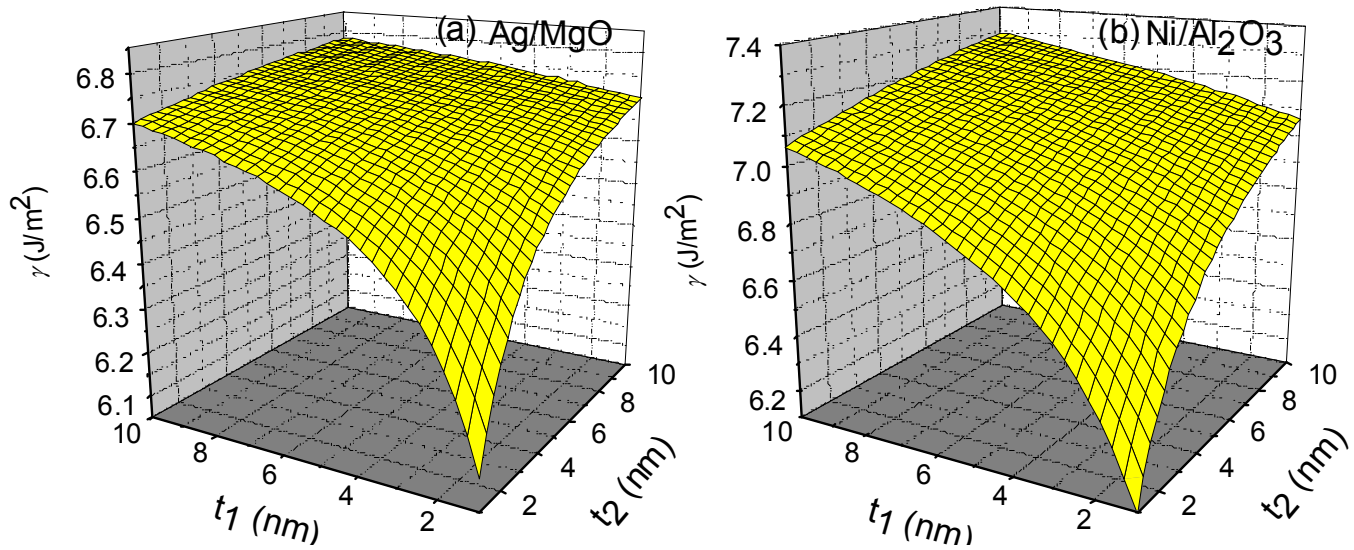

FIGURE 1. The calculated interface energy of $\mathrm{Ag} / \mathrm{MgO}$ (a) and $\mathrm{Ni} / \mathrm{Al}_{2} \mathrm{O}_{3}$ (b) in terms of Eq. (5). The related parameters in the equations are in TABLE 1 .

contribution to the total interface energy of the latter is larger. To understand the relation between the interface energy and the interface fracture properties, we compare the fracture strength of $\mathrm{Ag} / \mathrm{MgO}$ and $\mathrm{Ni}_{2} / \mathrm{Al}_{2} \mathrm{O}_{3}$ interfaces.

TABLE 1. The related parameters in the equations. For metals, $h=2 r$ with the atomic radius $r$. For ceramics, $V=M / \rho$ with the molar mass $M$ and the density $\rho, E=2 G(1+v)$ and $B=E /[3(1-2 v)] . h$ is taken as the average values of both elements for $\mathrm{MgO}$, and is taken as the bond length for $\mathrm{Al}_{2} \mathrm{O}_{3}$.

\begin{tabular}{lcccc}
\hline & $\mathbf{A g}$ & $\mathbf{N i}$ & $\mathbf{M g O}$ & $\mathbf{A l}_{\mathbf{2}} \mathbf{O}_{\mathbf{3}}$ \\
\hline$h(\mathrm{~nm})[6]$ & 0.3194 & 0.2754 & 0.3376 & $0.324[12]$ \\
$H(\mathrm{KJ} / \mathrm{mol})[7]$ & 11.3 & 17.47 & $90[9]$ & $111.4[13]$ \\
$T(\mathrm{~K})[7]$ & 1234 & 1726 & $3073[10]$ & $2326[13]$ \\
$V\left(\mathrm{~cm}^{3} / \mathrm{mol}\right)[7]$ & 10.3 & 6.59 & $11.26[10]$ & $25.49[13]$ \\
$G(\mathrm{GPa})[8]$ & 30.3 & 76 & $130[11]$ & $150[13]$ \\
$V[8]$ & 0.367 & 0.312 & $0.18[11]$ & $0.22[13]$ \\
$E(\mathrm{GPa})[8]$ & 82.7 & 199.5 & $307[11]$ & $366[13]$ \\
$B(\mathrm{GPa})[8]$ & 103.6 & 177.3 & $160[11]$ & $217.86[13]$ \\
\hline
\end{tabular}

Figure 2(a) shows the interface tension stress versus the interface tension displacement of $\mathrm{Ag} / \mathrm{MgO}$ systems based on the molecular mechanics calculation [14], it can be seen that the interface stress increases firstly with increasing interface tension displacement, then decreases after reaching the maximum stress, being consistent with the typical cohesive zone model describing the interface fracture [14]; the fracture strength, that is the maximum interface stress, is about 4.2 GPa. The shape of the stress-displacement curve of $\mathrm{Ag} / \mathrm{MgO}$ is similar to that of $\mathrm{Ni}_{2} / \mathrm{Al}_{2} \mathrm{O}_{3}$ interface as
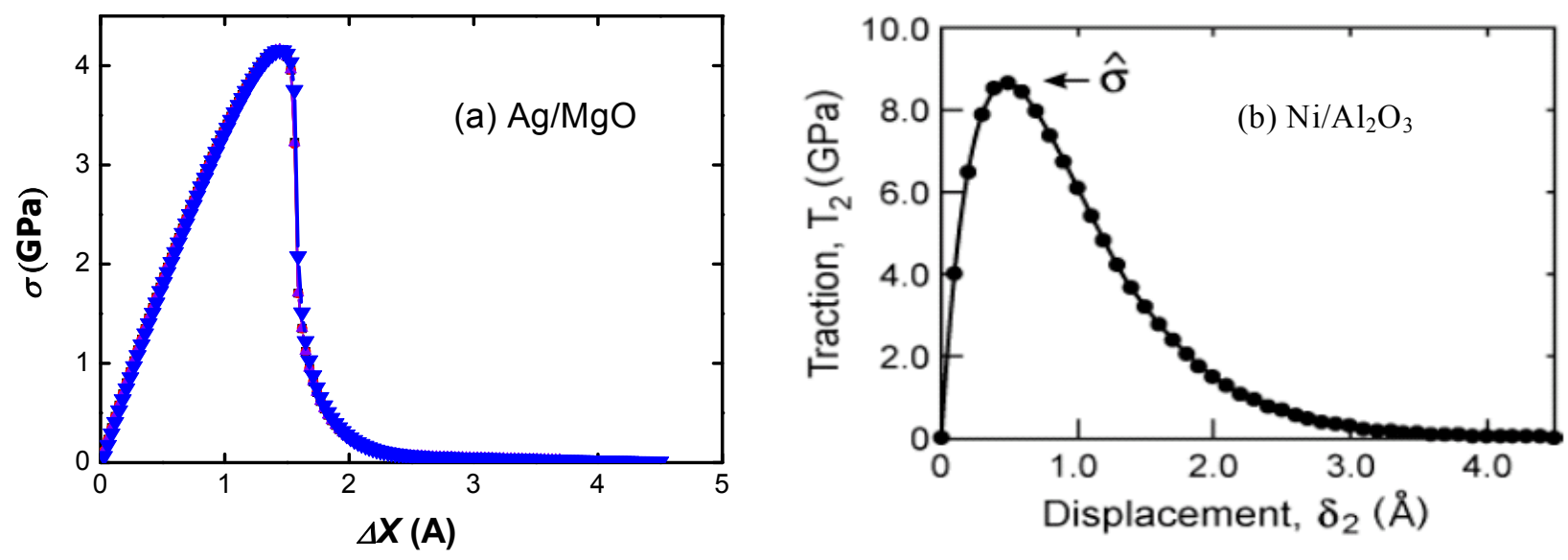

FIGURE 2. The interface stress versus the displacement of $\mathrm{Ag} / \mathrm{MgO}$ (a) and $\mathrm{Ni} / \mathrm{Al}_{2} \mathrm{O}_{3}$ (b). 
shown in Fig. 2(b), which is calculated based on the first principle [15]. Differently, the fracture strength of Ni/ $\mathrm{Al}_{2} \mathrm{O}_{3}$ interface is $8.3 \mathrm{GPa}$ [15], which is larger than that of $\mathrm{Ag} / \mathrm{MgO}$. Consequently, the fracture strength is larger for the interface with the higher total interface energy or with the lower chemical interface energy. It is understandable qualitatively that the fracture strength of the interface with the higher chemical interface energy is smaller since the interface is relatively unstable, at the same time, the smaller fracture strength corresponds to the lower total interface energy, i.e. the weaker interface adhesion ability, therefore, corresponds to the lower the structural interface energy, which indicates that the smaller strain work need to be overcome in the fracture process.

To understand quantitatively the relation between the interface energy and the interface fracture strength, we calculate the interface potential energy and the interface stress as the functions of the interface distance for $\mathrm{Ag} / \mathrm{MgO}$. The interface potential $\phi$ of $\mathrm{Ag} / \mathrm{MgO}$ refers to the modified Rahman-Stillinger-Lemberg potential as follows [16]

$$
\phi=a_{0} e^{d\left(1-\frac{x}{c_{0}}\right)}+\frac{a_{1}}{1+e^{b_{1}\left(x-c_{1}\right)}}+\frac{a_{2}}{1+e^{b_{2}\left(x-c_{2}\right)}}+\frac{a_{3}}{1+e^{b_{3}\left(x-c_{3}\right)}},
$$

where $x$ is the interface atomic distance, the other parameters are the potential parameters given by Chen-Mobius inversion method based on the ab initio interface adhesive energy calculation [16]. Considering there are two kinds of atomic pairs $\mathrm{Ag} / \mathrm{Mg}$ and $\mathrm{Ag} / \mathrm{O}$ across the interface, the potential parameters are all approximately taken as the average values of the both for the simplicity of the calculation. Figure 3 shows the calculated interface potential of $\mathrm{Ag} / \mathrm{MgO}$ based on Eq. (6), the absolute value of the minimum potential energy is $4.77 \mathrm{Kcal} / \mathrm{mol}$, corresponding to $6.3 \mathrm{~J} / \mathrm{m}^{2}$ after the unit conversion at $3.6 \mathrm{~nm}$ - the thickness of about four-layer metals and four-layer ceramics, which agrees well with the total interface energy at the same interface thickness calculated by Eq. (5) and implies that several nanometer is the appropriate size bridging the microscopic and macroscopic interface quantities.

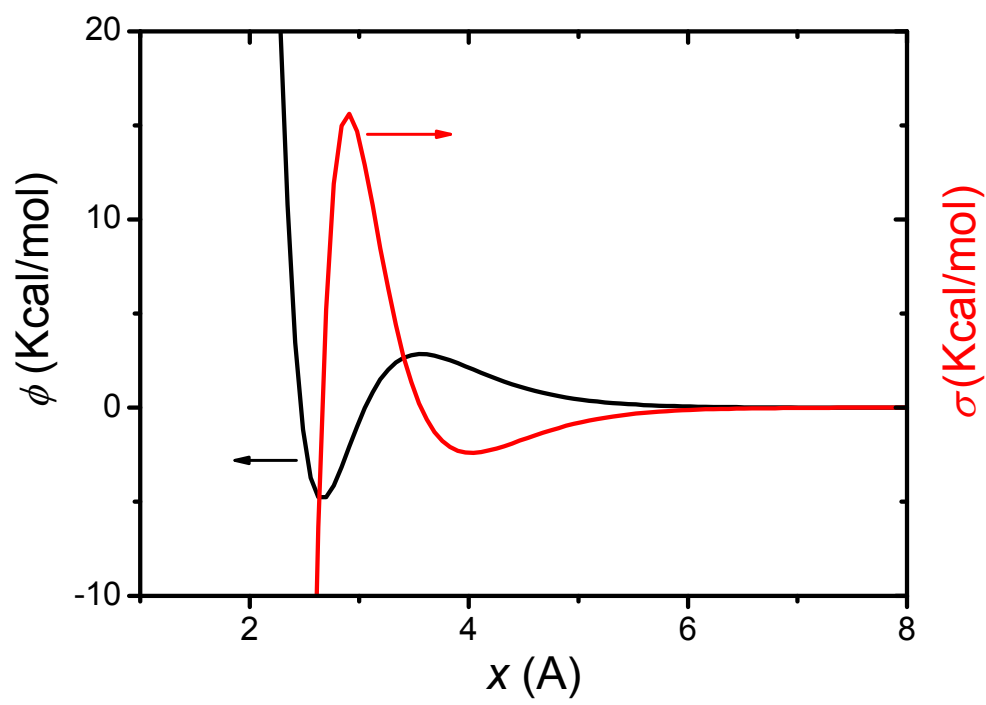

FIGURE 3. The interface potential and the stress versus the distance of $\mathrm{Ag} / \mathrm{MgO}$ in terms of Eq. (6). The potential parameters are in Table 1 in Ref. [16].

Furthermore, the interface stress as the first order derivative of the potential energy function is also shown in Fig. 3 , the maximum of the stress, i.e. the fracture strength, is $15.44 \mathrm{Kcal} / \mathrm{mol}$, that is about $5.7 \mathrm{GPa}$, which is close to the calculation result of the molecular mechanics as shown in Fig. 2(a). In fact, the calculation in Fig. 2(a) is based on the same interface potential, but is more accurate. In a word, the agreement proves that our model of the interface energy is reasonable. Moreover, the size effect of the interface energy at nanometer scale plays important role for bridging the microscopic and macroscopic interface quantities. 


\section{CONCLUSIONS}

In summary, an analytical model about nano-size dependence of the interface energy of metal/ceramic interfaces is developed by considering both the chemical component contribution and the structure strain contribution. The interface energy decreases as the thickness decreases, and the size effect depends on the atomic diameter, the melting enthalpy, the shear modulus and the Passion's ratio of metals and ceramics, etc. The comparison of the interface energy and the interface fracture strength of $\mathrm{Ag} / \mathrm{MgO}$ and $\mathrm{Ni} / \mathrm{Al}_{2} \mathrm{O}_{3}$ systems indicates that the fracture strength is larger for the interface with the higher total interface energy, the former corresponds to the maximum of the interface stress, and the latter corresponds to the absolute value of the minimum of the interface potential energy.

\section{ACKNOWLEDGMENTS}

The work is supported by the Research Grants from NSFC through grant nos. 10802088, 10832008, and The LNM initial funding for young investigators.

\section{REFERENCES}

1. Q. Jiang, D. S. Zhao, M. Zhao, Acta. Mater. 49, 3143-3147, (2001).

2. G. Ouyang, L. H. Liang, C. X. Wang, G. W. Yang, Appl. Phys. Lett. 88, 091914, (2006).

3. Kotze, D. Kuhlmann-Wilsdorf, Appl. Phys. Lett. 9, 96, (1966).

4. R. C. Cammarata, K. Sieradzki, F. Spaepen, J. Appl. Phys. 87, 1227-1234, (2000).

5. Q. Jiang, L. H. Liang, D. S. Zhao, J. Phys. Chem. B 105, 6275-6277, (2001).

6. H. W. King, Physical Metallurgy, edited by R. W. Cahn, Amsterdam: North-Holland Pub. Co., p. 64, (1983).

7. Periodic Table of the Elements, Skokie, Illinois: Sargent-Welch Scientific Company, p. 1, (1980).

8. Smithells Metals Reference Book, edited by E. A. Brandes, London: Butterworth \& Co Ltd., p. 15-2, (1983).

9. R. E. Cohen, J. S. Weitz, arxiv.org/abs/mtrl-th/9605001, (3 May 1996).

10. http://en.wikipedia.org/wiki/Magnesium_oxide.

11. C. S. Zha, H. K. Mao, R. J. Hemley, Proceedings of the National Academy of Sciences of the United States of America, 10.1073/pnas.240466697, (28 November 2000 online).

12. http://www.qivx.com/ispt/elements/ptw_013.php.

13. http://www.matweb.com/search/DataSheet.aspx?MatGUID=c8c56ad547ae4cfabad15977bfb537f1.

14. X. M. You, "The study on the microscopic mechanism of the cohesive zone model of Ag/MgO interface", Ph.D. Thesis, Institute of Mechanics, Chinese Academy of Sciences, (2008).

15. J. R. Smith, Y. Jiang, A. G. Evans, Int. J. Mat. Res. 98, 1214, (2007).

16. Y. Long, N. X. Chen, W. Q. Zhang, J. Phys.: Condens. Matt. 17, 2045-2058, (2005). 